\title{
Manipulation of a quasi-natural cell block for high-efficiency transplantation of adherent somatic cells
}

\author{
H.J. Chung ${ }^{1 *}$, M.M. Hassan ${ }^{1 *}$, J.O. Park ${ }^{1}$, H.J. Kim² and S.T. Hong ${ }^{1}$ \\ ${ }^{1}$ Department of Biomedical Sciences, Institute for Medical Science, \\ Chonbuk National University Medical School, Jeonju, Chonbuk, South Korea \\ ${ }^{2}$ JINIS BDRD Institute, JINIS Biopharmaceuticals Co., Wanju, Chonbuk, South Korea
}

\begin{abstract}
Recent advances have raised hope that transplantation of adherent somatic cells could provide dramatic new therapies for various diseases. However, current methods for transplanting adherent somatic cells are not efficient enough for therapeutic applications. Here, we report the development of a novel method to generate quasi-natural cell blocks for high-efficiency transplantation of adherent somatic cells. The blocks were created by providing a unique environment in which cultured cells generated their own extracellular matrix. Initially, stromal cells isolated from mice were expanded in vitro in liquid cell culture medium followed by transferring the cells into a hydrogel shell. After incubation for 1 day with mechanical agitation, the encapsulated cell mass was perforated with a thin needle and then incubated for an additional 6 days to form a quasi-natural cell block. Allograft transplantation of the cell block into C57BL/6 mice resulted in perfect adaptation of the allograft and complete integration into the tissue of the recipient. This method could be widely applied for repairing damaged cells or tissues, stem cell transplantation, ex vivo gene therapy, or plastic surgery.
\end{abstract}

Key words: Quasi-natural cell block; Transplantation; Scaffold; Extracellular matrix; Hydrogel

\section{Introduction}

Replacement of damaged tissues or organs with somatic cells is currently a major objective of tissue engineering. However, applications of cell transplantation are not confined to tissue engineering; recent studies have described therapeutic applications for transplantation of unmodified adherent stem cells $(1,2)$. Many experimental animal studies and clinical trials have shown that transplantation of adult or embryonic adherent stem cells can improve various degenerative diseases such as diabetes, Alzheimer's disease, and Parkinson's disease, among others. $(3,4)$. Although cell transplantation-based treatments are making impressive progress, the efficiency of current cell transplantation methods is not high enough to achieve therapeutic goals. Therefore, the development of new, more efficient methods is a major scientific challenge in the fields of tissue engineering and stem cells.

Mammals have a closed cardiovascular system, which limits the movement of blood cells out of the network of arteries, veins and capillaries. Consequently, intravenously injected adherent somatic cells continue circulating until they self-destruct or are removed by the immune system of the recipient, and only rarely do they survive in the recipient. Intravenously injected adherent somatic cells occasionally block blood vessels, leading to deleterious consequences (5). In this context, simple intravenous injection of adherent somatic cells could not be a solution for achieving cell transplantation. To overcome problems of cell transplantation by intravenous injection of adherent somatic cells, scaffold-based approaches have been widely adopted in the fields of tissue engineering and stem cells for transplantation of adherent somatic cells. In the scaffold-based cell transplantation approaches, the adherent somatic cells are mixed with porous biocompatible materials, and the mixture is transplanted into the recipient (Figure 1A). If necessary, the adherent somatic cells are

Correspondence: Seong-Tshool Hong: <seonghong@chonbuk.ac.kr>.

${ }^{*}$ These authors contributed equally to this study.

The present address of J.O Park is Bone Marrow Transplantation Division, School of Medicine, University of Louisville, Louisville, KY 40292, USA.

Received August 14, 2014. Accepted November 10, 2014. First published online March 3, 2015. 


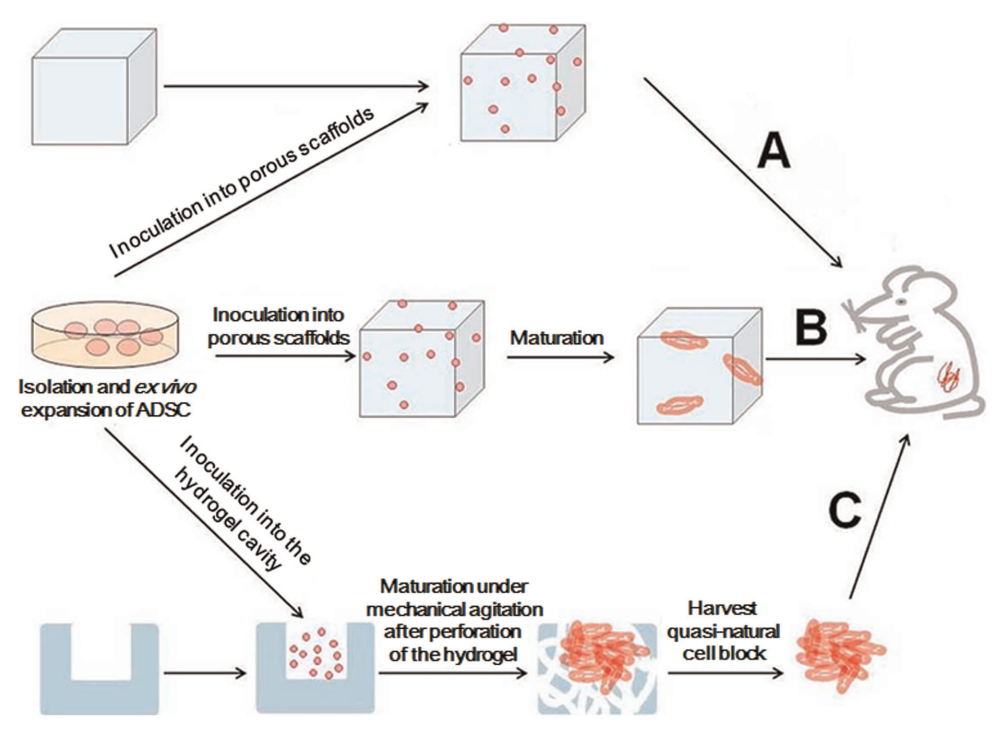

Figure 1. Schematic diagram of strategies to transplant adherent somatic cells. $A$, Transplantation of adherent somatic cells based on a mixture approach. $B$, Transplantation of adherent somatic cells by $3 \mathrm{D}$ culture. $C$, Transplantation of adherent somatic cells through manipulation of the quasinatural cell block.

cultured in the mixture to grow and to mature into adherent somatic cells (Figure 1B). A variety of scaffold materials have been developed, ranging from soft materials such as hydrogels to hard, amorphous thermoplastics such as polystyrene (6-9). Cells embedded in the 3-dimensional (3D) scaffold are expected to multiply, migrate, secrete signals, and eventually generate extracellular matrix (ECM) (10). In the scaffold-based cell transplantation process, cells within the scaffold materials are entirely responsible for synthesizing tissue constituents and for assembly of functional tissue subunits $(11,12)$. Ironically, the scaffold materials have been the root of problems encountered in this transplantation process. In mixtures of cells and scaffold material, the quantity of scaffold material needs to exceed that of cells to support structural integrity, which means that wide spatial separation between cells cannot be avoided in the scaffoldbased cell transplantation process. Because of the intervening distance between the cells embedded in the scaffold materials, cellular stimuli and/or interaction with other cells that are required for the proper growth, maturation, and formation of an ECM and appropriate tissue microarchitecture are limited (13). The problems resulting from spatial separation between cells cannot be resolved completely, even in the case of biodegradable scaffolds such as hydrogels $(14,15)$. Therefore, various approaches such as mechanical agitation and supplementation with growth factors and/or adhesion molecules have been used to overcome the spatial separation problem and to provide a more favorable environment (16). However, in practice none of these modified scaffold-based cell transplantation methods were satisfactory for the practical application of cell transplantation in tissue engineering and stem cell therapy.

In this study, we developed a method to create a quasinatural cell block for high efficiency transplantation of adiposederived mesenchymal stromal cells (ADMS) (Figure 1C). ADMS isolated from the adipose tissue of mice were expanded in vitro, and then the cultured ADMS cells were transferred into a precast cavity in a hydrogel. The ADMS cells were allowed to grow and mature in the hydrogel. In this chemically and physically unique environment, they generated an ECM and were transformed into a quasi-natural cell block. Following transplantation into a recipient, the cell blocks became well adapted, showing biological characteristics that were almost identical to the connective tissue typical of the recipient. This quasi-natural cell block might be used not only in cell transplantation but also as a module for creation of artificial tissues biologically identical to the real tissue in the body.

\section{Material and Methods}

\section{Animal experiments}

Six-week-old male and female C57BL/6 mice (Joongang Experimental Animal Co., Korea) were purchased and acclimatized for 2 weeks before being used in the experiments. When the study procedures were conducted, the mice weighed $20-24 \mathrm{~g}$ and were 8-12 weeks of age. This study was carried out in strict accordance with the guidelines of the Ethics Committee of Chonbuk National University Laboratory Animal Center, which approved the protocol (Permit No. CBU 2012-0040). All efforts were made to minimize suffering.

\section{Isolation and ex vivo expansion of ADMS cells}

Adipose tissue was surgically obtained from the abdominal region of male mice and processed for ADMS culture as follows. The tissue was cut into small pieces and enzymatically digested with $0.2 \%$ collagenase (Sigma, USA) in phosphate buffered saline (PBS) for $1 \mathrm{~h}$ at $37^{\circ} \mathrm{C}$ with gentle agitation. The collagenase was inactivated with an equal volume of Dulbecco's Modified Eagle's Medium (DMEM; HyClone, USA) supplemented with $10 \%$ fetal bovine serum 
(FBS, HyClone) and centrifuged at $400 \mathrm{~g}$ for $5 \mathrm{~min}$ at room temperature. The resulting cell pellet was suspended in $0.83 \% \mathrm{NH}_{4} \mathrm{Cl}$, incubated for 2 min to eliminate red blood cells and passed through a 100- $\mu \mathrm{m}$ mesh filter (BD Biosciences, USA) to remove cell aggregates and connective tissue debris. The cells were then collected by centrifugation at $400 \mathrm{~g}$ for $5 \mathrm{~min}$ and the pellet was suspended in Mesencult ${ }^{\mathrm{TM}}$ medium (Stemcell Technologies, Canada) supplemented with mesenchymal stem cell stimulatory supplements (Stemcell Technologies), and plated in collagencoated $175 \mathrm{~cm}^{2}$ cell culture flasks (T175; BD Biosciences, USA). ADMS cells were maintained at $37^{\circ} \mathrm{C}$ in a $5 \% \mathrm{CO}_{2}$ atmosphere. After $12-16 \mathrm{~h}$, the nonadherent cells were removed and adherent cells were cultured for further expansion. At $70-80 \%$ confluence, they were trypsinized and subcultured at a density of $5 \times 10^{3}$ cells $/ \mathrm{cm}^{2}$ in T175 flasks for use in tissue engineering. The doubling time of ADMS cells in log phase was determined by the Patterson equation (17). The growth kinetics of ADMS cells was determined at passage six by the methylthiazol-diphenyltetrazolium (MTT) assay (Sigma) according to the manufacturer's instructions. All experiments and measurements were carried out at least in triplicate.

\section{Preparation of quasi-natural cell blocks}

Matrige $^{\mathrm{TM}}$ (BD Biosciences) was thawed overnight at $4^{\circ} \mathrm{C}$, a homogenous mixture was formed by gentle pipetting, and $100 \mu \mathrm{L}$ of the gel was pipetted into each well of 24-well plates and maintained at $37^{\circ} \mathrm{C}$ for $30 \mathrm{~min}$ to solidify. Each well contained a T-shaped glass rod in the center, which was then removed, leaving a cavity in the hydrogel. Fifty microliters of ADMS cells suspended in PBS $\left(6 \times 10^{6}\right.$ cells $/ \mathrm{mL}$ ) were poured into the hydrogel cavity, and then $20 \mu \mathrm{L}$ of the gel was layered on top of the cell mass in the hydrogel cavity. The cell mass, completely surrounded by the hydrogel shell, was then transferred to a petri-dish containing $10 \mathrm{~mL}$ Mesencult ${ }^{\mathrm{TM}}$ medium and incubated at $37^{\circ} \mathrm{C}$ in a $5 \% \mathrm{CO}_{2}$ atmosphere for 1 day with gentle shaking at 10 rpm on an orbital shaker. Following 1 day of maturation, the hydrogel-encapsulated cell mass was perforated several times with a thin, 27-gauge needle. The perforated cell mass was incubated again at $37^{\circ} \mathrm{C}$ in a $5 \% \mathrm{CO}_{2}$ atmosphere for an additional 6 days on the orbital shaker at $10 \mathrm{rpm}$ to form the quasi-natural cell block. The blocks were then harvested by removing the hydrogel shells with a spatula followed by incubation in dispase solution (Stemcell Technologies) at $37^{\circ} \mathrm{C}$ for $15 \mathrm{~min}$ to remove excess hydrogel. The blocks were then washed 3 times in PBS before implantation. The quasi-natural cell blocks were transplanted subcutaneously into 8-week-old C57BL/6 female mice weighing 20-24 g and anesthetized with Zoletil $50^{\circledR}$ (Virbac, USA), and then ligated with a 5.0 silk suture (Ethicon, USA).

\section{Histological examination}

The transplanted cell blocks were removed by dissection after sacrificing the mice by $\mathrm{CO}_{2}$ inhalation. For microscopic evaluation, the tissue was fixed in $10 \%$ neutral buffered formalin for $24 \mathrm{~h}$ and embedded in paraffin. The paraffinembedded tissue was sectioned at $5 \mu \mathrm{m}$ and mounted on silanized slides by floating the sections on water. The slides were then air-dried and held overnight at $65^{\circ} \mathrm{C}$. For histochemical analysis, the deparaffinized tissue sections were stained with hematoxylin and eosin (H\&E) and were examined by light microscopy. For immunohistochemistry of the tissue sections, slides were placed into a staining jar (Kartell, Italy) filled with Retrievagen A solution (BD Biosciences). The jar was placed on a turntable plate in a microwave oven and heated at $100 \mathrm{~W}$ for $5 \mathrm{~min}$ for three cycles. After cooling to room temperature, the slides were washed and incubated with $0.16 \%$ trypsin for $10 \mathrm{~min}$ at $37^{\circ} \mathrm{C}$ and further washed with PBS. The slides were then fixed in $4 \%$ paraformaldehyde for $2 \mathrm{~min}$, dehydrated in an ethanol series to $100 \%$ ethanol, and blocked with 10\% normal rabbit and goat serum in PBS for $1 \mathrm{~h}$. The blocked sections were stained overnight at $4{ }^{\circ} \mathrm{C}$ with rabbit anti-mouse connexin43, rabbit anti-mouse vimentin, goat anti-mouse cytokeratin and rabbit anti-mouse CD31 (Invitrogen, USA). Secondary AlexaFluor-488-conjugated goat anti-rabbit lgG and Alexa-Fluor568-conjugated rabbit anti-goat IgG antibodies (Invitrogen) were used for detection. Confocal images were obtained with an LSM510 Meta microscope (Carl Zeiss, Germany).

\section{Fluorescence in situ hybridization (FISH)}

For FISH analysis, slides were treated with $1 \mathrm{M}$ sodium thiocyanate for $10 \mathrm{~min}$ at $80^{\circ} \mathrm{C}$, rinsed in PBS, and heated at $100 \mathrm{~W}$ for $5 \mathrm{~min}$ for three cycles in a microwave oven with a turntable plate. After cooling to room temperature, the slides were washed, digested with pepsin solution (ID Labs, USA) for $15 \mathrm{~min}$ at $37^{\circ} \mathrm{C}$, quenched in glycine solution, and washed with PBS. After fixation and dehydration in ethanol, slides were stained with Y-paint FISH probe (ID Labs) and then washed with $50 \%$ formamide solution in $2 \times$ salinesodium citrate (SSC) buffer, with $2 \times$ SSC, and with $4 \times$ SSC. The slides were counterstained with 4',6-diamidino-2phenylindole (DAPI) solution (ID Labs) and examined using a Carl Zeiss LSM510 Meta microscope.

\section{Results}

A quasi-natural cell block was created by providing a unique environment in which cells grew by generating their own extracellular matrix

Although any type of adherent somatic cell can be used to construct quasi-natural cell blocks, ADMS cells were chosen for this study because the simple transplantation of ADMS cells has been reported to be effective for treatment of a wide range of diseases (18). ADMS cells are multipotent and able to differentiate along multiple lineage pathways (19). More important, they can be obtained easily and in abundance by a minimally invasive procedure. ADMS cells were isolated from peritoneal adipose tissue excised from the abdominal region of male C57BL/6 mice. For in vitro 

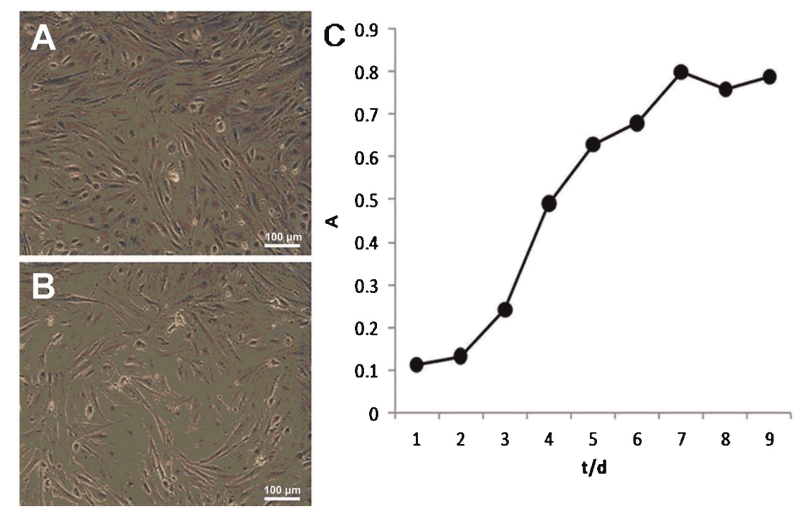

Figure 2. Preparation of adipose-derived mesenchymal stromal (ADMS) cells to manipulate the quasi-natural cell block. ADMS cells were isolated from peritoneal adipose tissue excised from the abdominal region of male C57BL/6 mice and cultured for in vitro expansion. Mouse ADMS cells at passage $2(A)$ and passage $6(B)$ showed similar fibroblast-like morphology. The growth curve of ADMS cells was determined at passage 6 by using the MTT assay $(C)$; an initial lag phase of 2 days, a log phase from day 3 to day 5 , and a plateau phase.

expansion of ADMS cells, primary culture was carried out after enzymatic digestion with collagenase, followed by centrifugation at $400 \mathrm{~g}$ for $5 \mathrm{~min}$ and washing. After two or more successive passages in culture, ADMS cells are known to express characteristic adhesion and receptor molecules, surface enzymes, extracellular matrix and cytoskeletal proteins, and proteins associated with the stromal cell phenotype. The cells were passaged every 4-5 days at about $80 \%$ confluence for 12 passages. During the culture period, there were no morphologic alterations. Typical primary or passaged ADMS cells displayed fibroblast-like morphological features (Figure 2A and B). The proliferation capacity and senescence of cultured ADMs cells were analyzed because the proliferative ability of cells is important with regard to their use in cell therapy and tissue engineering (20). The growth kinetics of ADMS cells was determined at passage 6 by MTT assay. The growth curves displayed an initial lag phase of 2 days, a log phase from day 3 to day 5 , and a plateau phase (Figure $2 \mathrm{C}$ ). According to the Patterson equation, the doubling time in the log phase was $33.1 \pm$ $1.4 \mathrm{~h}(\mathrm{P}>0.05)$, implying high proliferation capacity without any sign of senescence.

Matrige $^{\mathrm{TM}}$, a type of hydrogel, is one of the most widely used natural materials in tissue engineering (21). To generate quasi-natural cell blocks in vitro, Matrigel ${ }^{\mathrm{TM}}$ was used as a casting scaffold added to the in vitro ADMs cultures. Matrigel $^{\mathrm{TM}}$ was precast to form a cavity into which ADMS cells were transferred. The hydrogel-encapsulated cells were incubated under mechanical agitation and allowed to mature in the hydrogel cavity for 1 day. The hydrogel was then perforated several times with a thin needle and incubated further for 6 days to form a quasi-natural cell block (Figure 3A). After 6 days of tissue growth within the

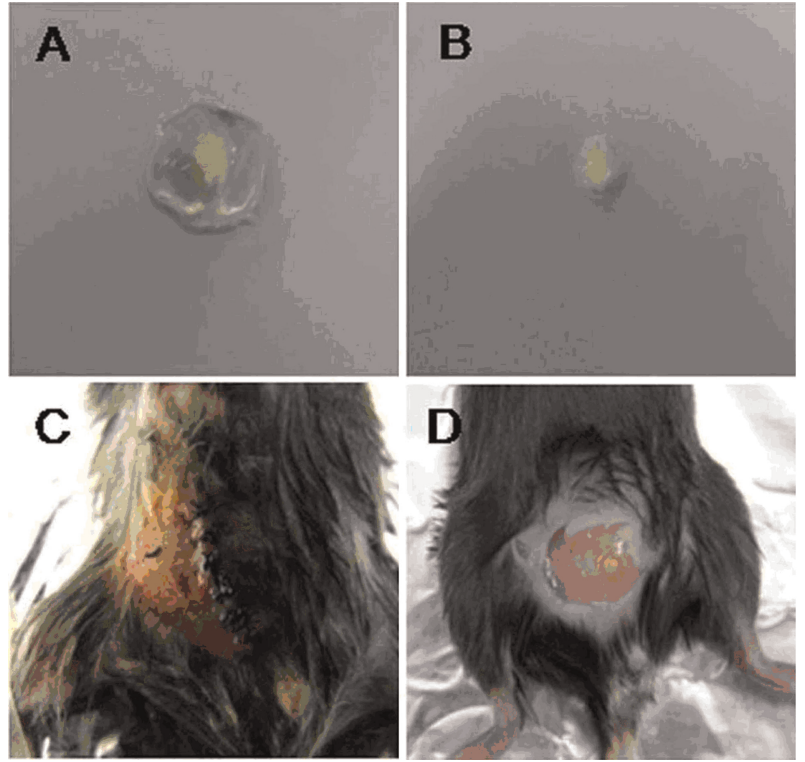

Figure 3. In vitro construction and implantation of the quasinatural cell block. The quasi-natural cell block cultured for 7 days $(A)$ was harvested by removing its surrounding Matrigel ${ }^{\mathrm{TM}}$-cast for transplantation $(B)$. The quasi-natural cell block was implanted into subcutaneous tissue of a female mouse $(C)$ and allowed to adapt in vivo for 4 weeks, showing successful connection with the surrounding tissue of the recipient $(D)$.

perforated hydrogel cavity, the quasi-natural cell block was removed (Figure 3B).

\section{The quasi-natural cell block dramatically improved transplantation efficiency of ADMS cells compared with conventional implants}

After successful manipulation of quasi-natural cell blocks, they were implanted into the subcutaneous tissue of female C57BL/6 mice (Figure 3C) and allowed to regenerate for 4 weeks (Figure 3D) to confirm the transplantation efficiency of ADMS cells. Because the transplantation efficiency of adherent somatic cells following simple intravascular or subcutaneous injection is very poor, they are mixed with scaffolding materials such as a hydrogel for transplantation in most conventional methods (22). Frequently, the scaffolding material and adherent somatic cells are cultured together in vitro before transplantation (23). Morphological and histological examinations of ADMS cells that were transplanted as quasi-natural cell blocks were conducted in parallel with the evaluation of transplants of conventional mixtures of a hydrogel and ADMS cells (Figure 4). H\&E staining showed that most of the space in the conventional mixtures in vitro was occupied by the scaffold material itself rather than cells (Figure 4A), resulting in local clustering of cells in vivo after implantation (Figure 4B). This occurred primarily because the hydrogel must make up at least $70 \%$ of the mixture volume to maintain the solid structure in vitro. Thus, the majority of substance of the artificial tissues was 

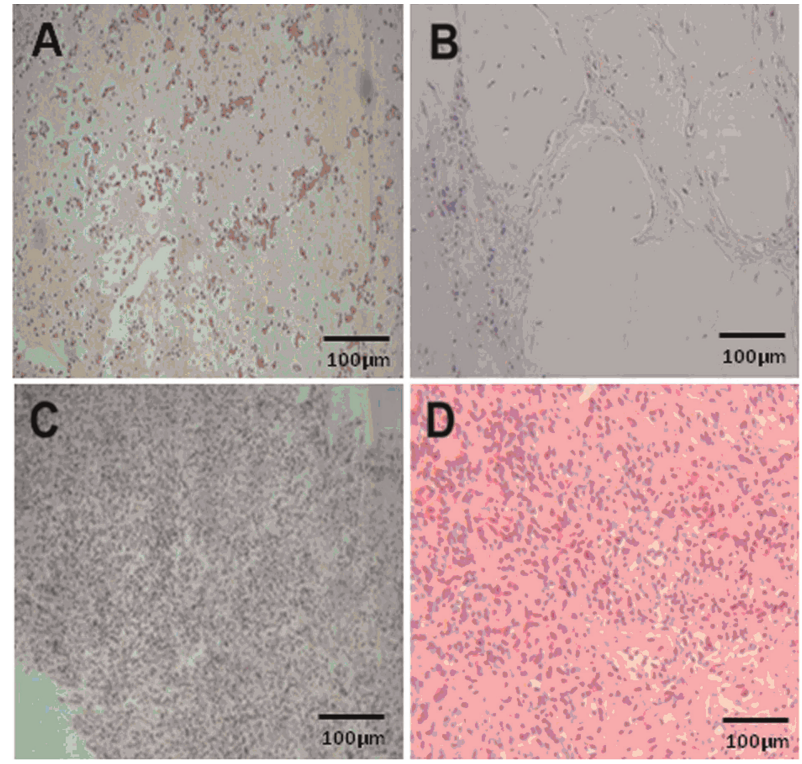

Figure 4. Evaluation of the quasi-natural cell block. ADMS cells were mixed with Matrigel ${ }^{\mathrm{TM}}$ gel to transplant the mixture in a conventional approach and tissue sections were prepared for histochemical staining $(A, B)$. The quasi-natural cell block $(C, D)$ was manipulated and histologically evaluated in comparison with the conventional method. In the conventional method, H\&E staining showed mostly scaffold materials with a few encapsulated cells in the mixture before transplantation $(A)$ and after transplantation $(B)$. In the quasi-natural cell block, however, much higher densities of ADMS cells were observed before transplantation $(C)$ as well as after transplantation $(D)$.

scaffold itself rather than the network of ADMS cells. In fact, improper aggregation of cells and excessive space occupied by the scaffold material would inhibit blood circulation after transplantation of ADMS cells, resulting in cell damage. Damaged cells initiate inflammation, which attracts immune cells to the transplanted tissue, and the immune reaction damages all of the transplanted tissue $(24,25)$.

In contrast, histological sections of quasi-natural cell blocks in vitro revealed much higher densities of ADMS cells than in the conventional mixtures (Figure 4C). Consequently in vivo, the quasi-natural cell blocks included more ADMS cells, which were evenly distributed and had cellular connections (Figure 4D) resembling typical subcutaneous tissue. In the quasi-natural cell blocks, the presence of clusters of cells without exogenous scaffold material, just as in native tissue, might promote interactive communication among neighboring cells, which could enhance cell viability as well as functional regeneration of implanted tissues.

\section{Quasi-natural cell blocks were adapted perfectly in the recipient and become completely integrated into the recipient tissue}

A major defect of conventional cell transplantation methods using mixtures of cells and scaffold materials is the lack of functional vasculature and connection with surrounding tissues, which leads to cellular necrosis and failed regeneration of bioengineered tissue in the recipient body (26). As shown in Figure $5 \mathrm{~A}-\mathrm{C}, \mathrm{H} \& \mathrm{E}$ staining of ADMS cells transplanted using the conventional mixture approach showed that only a few transplanted ADMS cells survived in vivo. However, transplantation of quasi-natural cell blocks was followed by extensive proliferation of ADMS cells and formation of vascular networks (Figure 5D and E). In addition to the vasculature, morphological examination of the transplanted quasi-natural cell blocks also revealed satisfactory differentiation of ADMS cells into various cell types, including fibroblasts, adipocytes, and multinucleated giant cells, as well as formation of capillary vessels with erythrocytes (Figure 5E-I)

Taken together, the results demonstrated successful in vivo implantation of the quasi-natural cell blocks created in this study. The transplanted cells were further investigated using specific cell-lineage markers to confirm the complete adaptation and maintenance after implantation (Figure 6). To confirm the transplantation efficiency through allogenicity, gender-specific probes were used to distinguish the male-derived allografted tissue from the recipient female mouse by FISH, along with DAPI staining of the nucleus (Figure $6 \mathrm{~A}$ and $\mathrm{B}$ ). The $\mathrm{Y}$ chromosome-specific probe detected large numbers of male lineage nuclei in the allografted area in the recipient female mice. There were no cells in the vacant space surrounding the tissue, indicating that the transplanted tissues were comprised of male ADMS cells and were well adapted to become a part of the connective tissue. The potential for intercellular communication in the allografted tissue was checked with connexin43, which is a gap junction protein with a great impact on cell-to-cell communication within tissues (27). Immunofluorescence staining of allografted tissue specimens compared with native tissues showed that the connexin43 protein was similarly distributed in both (Figure 6C), and indistinguishable from native heart tissue samples (Figure 6D). The uniform distribution of connexin43 protein throughout the allografted quasinatural tissue indicated that the cells within the tissue were able to maintain proper communication and signaling. Immunostaining of the allografted quasi-natural tissue also revealed evidence of multiple lineages, including endothelial lineage (anti-CD31) and mesodermal lineage (antivimentin) markers, without detection of an epithelial lineage marker (anticytokeratin). CD31, a representative marker of endothelial cells, is clearly shown in allografted quasi-natural tissue with vascular network formation in Figure 6E. Vimentin is a cytoskeletal component responsible for maintaining cell integrity, and the detection of vimentin, but not cytokeratin, in the allografted quasinatural tissue, indicates the formation of structural, cytoskeletal networks for connective tissue (Figure 6F). This implies that the allografted ADMS cells differentiated into endothelial cells, which participate in the formation of 


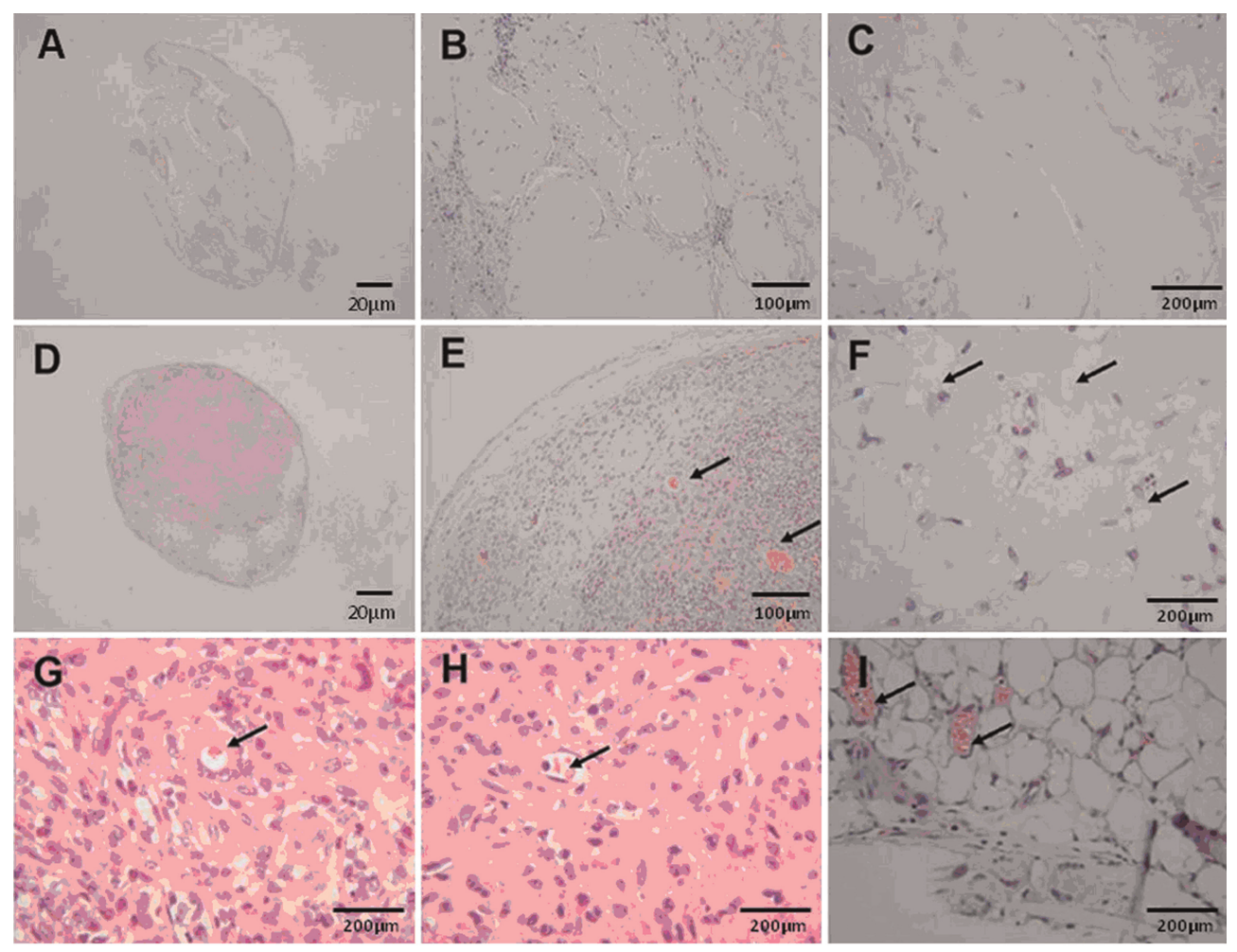

Figure 5. Successful regeneration of the quasi-natural cell block in vivo. H\&E staining showed small numbers of the transplanted ADMS cells in the conventional mixture approach as well as a general unhealthy shape of the transplanted cells $(A-C)$. In contrast, the quasi-natural cell block implants displayed successful proliferation of implanted ADMS cells $(D, E)$. Morphological examinations further revealed differentiation of ADMS cells into various cell types as well as formation of capillary vessels with erythrocytes $(E-I)$. Arrows indicate erythrocytes $(\mathrm{E}, \mathrm{G})$, adipocytes $(F)$, or multinuclear giant cells $(H, I)$.

vessels, confirming that epithelialization of the epidermis and tissue regeneration had occurred in the allografted quasi-natural tissue.

\section{Discussion}

The high demand in modern medicine for transplantation of cells or tissues has been a driving force to develop highly efficient transplantation methods. Although the mixing of cells with 3D, porous biocompatible materials has been the most widely adopted approach for cell transplantation, it has not been successful because it is not highly efficient $(28,29)$. The main reason for the failure of that approach is based on the intrinsic difficulties in the development of complete ECM mimics using scaffolds. In natural tissues, cells interact with each other to create an ECM that dictates the morphological and physical changes in cells that are needed for high efficiency transplantation. Recent evidence strongly indicates that the ECM is not only a determining factor for cell differentiation in tissue but is also responsible for the proper biochemical functioning of the tissue cells $(30,31)$. In 3D porous biocompatible scaffolds such as hydrogels, scaffold materials have to comprise at least $70 \%$ of the cell mixture to maintain the structural rigidity needed for growth of artificial tissues. This results in a low density of cells in the mixture and consequently a large spatial separation among cells, making it difficult for them to communicate. Moreover, the scaffolds cannot mimic the complexity of ECM architectures formed naturally by cells in real tissues, which is essential for proper growth and development of transplanted cells into tissues $(32,33)$. Therefore, it would be unrealistic to expect that high efficiency transplantation of cells is possible using current methods based on the simple mixing of cells and scaffold materials, or growing cells within a 3D porous biocompatible scaffold.

We successfully created a quasi-natural cell block that is biologically indistinguishable from the recipient tissue. Histological evaluation of transplanted cell blocks showed that well-clustered cell lines were able to maintain proper intercellular communication and an ECM, assemble a vascular network, and adapt permanently after engraftment without immunogenicity. As shown in Figure 3, the transplanted quasi-natural cell block was perfectly adapted in the recipient. The adapted tissues that originated from the quasi-natural cell block were histologically indistinguishable from the neighboring tissues of the recipient mice (Figure 5). 

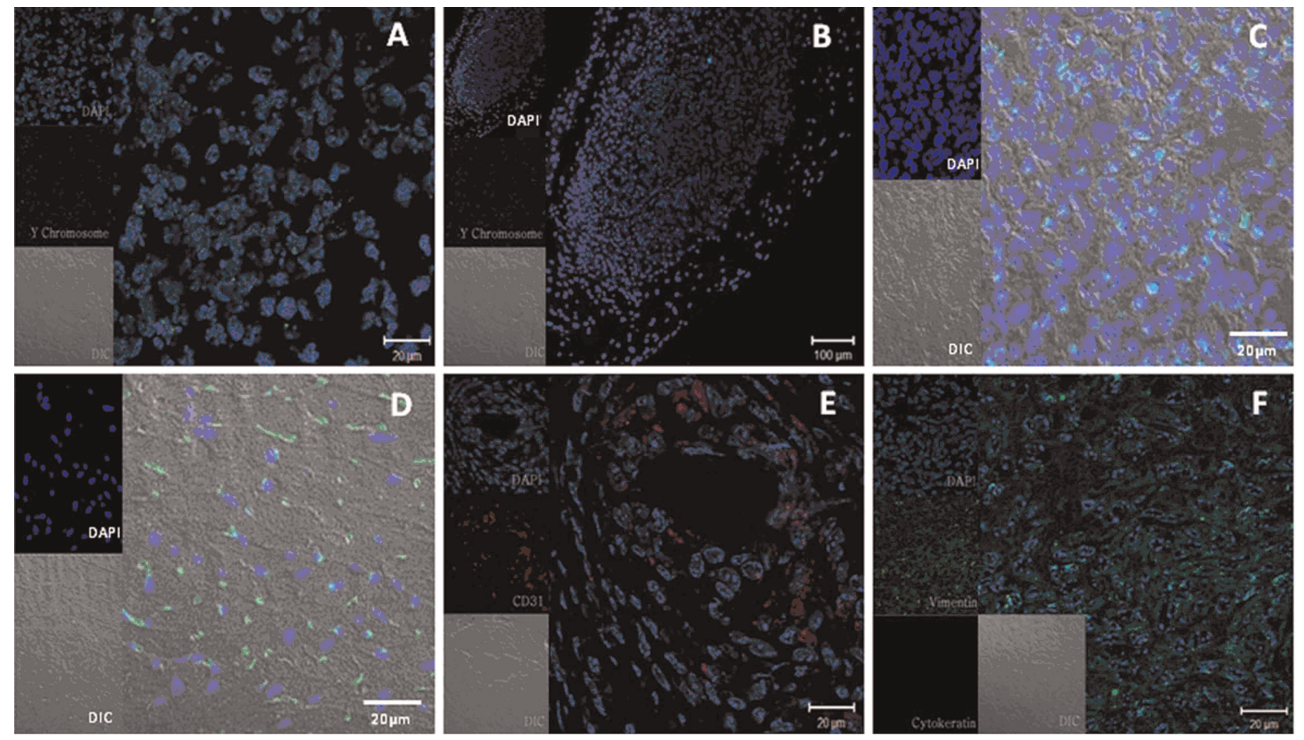

Figure 6. Confirmation of the allografted quasi-natural cell block in the recipient body. To confirm allogenicity of the transplanted quasinatural cell block, gender-specific probes were used to distinguish the male-derived allografted tissue from the recipient female mouse by FISH along with DAPI staining of the nucleus $(A, B)$. Green spots indicate male $Y$ chromosome probe in the corresponding female tissue. For intercellular communication capability in the allografted tissue, specimens were stained with specific probes for signaling marker connexin43, in the quasi-natural tissue $(C)$ along with native heart tissue $(D)$. Immunostaining of the allografted quasi-natural tissue with epithelial linage marker CD31 revealed vascular network assembly as indicated $(E)$. Immunostaining of the allografted quasi-natural tissue further showed that most of cells were stained with anti-vimentin, mesodermal lineage marker, but not with anticytokeratin $(F)$. Images for individual channels (connexin43 with alexa 488 is green, cd31 with alexa 568 is red, vimentin with alexa 488 is green, cytokeratin with alexa 568 is red) are shown on the left, and main panels show the merged image containing all channels plus DIC. The cells nuclei were visualized with DAPI (blue). Scale bar: $(A, C, D, E$ and F) $20 \mu \mathrm{m} ;(B) 100 \mu \mathrm{m}$.

The formation of blood vessels that was observed in the transplant indicates that the quasi-natural cell block became connective tissue of the recipient. Since the transplanted cell block tissue was almost indistinguishable from that of the neighboring tissues in the recipient mice by histological examination, it was possible to mistakenly identify some tissue of the recipient mice as transplanted, quasi-natural tissue. However, this possibility was clearly excluded by the in situ hybridization with a $\mathrm{Y}$-chromosome probe that was clearly positive only in the male tissue cells from the quasi-natural cell block present in the female mouse body (Figure 6).

Our method of transplanting adherent somatic cells is intrinsically different from previous approaches, which combine cells and a biocompatible 3D scaffold prior to transplantation. Sometimes the cells are grown to maturity before being combined with the 3D scaffold for transplantation (34). However, none of the previous approaches has resulted in satisfactory, high-efficiency transplantation of cells. In this approach, we showed that the cells growing and maturing in a 3D environment while generating their own ECM created a tissue block that became biologically indistinguishable from the body tissue of the recipient. The ECM formed naturally from collagens secreted by neighboring cells and was similar to the ECM of the body tissues. This ECM supported and maintained proper intercellular communication, allowing ADMS cells to become a part of the surrounding tissue in the recipient. Our work exemplifies the importance of natural ECM during transplantation of cells.

The vasculature provides an effective delivery system for transport of cellular nutrients, but the current cell transplantation methods based on mixtures of cells and biocompatible scaffold materials lacks such a mechanism. The resulting dependence on passive mass transport results in poor cell transplantation efficiency (35). Transport of oxygen and nutrients in 3D culture depends on passive diffusion, and is typically considered as the main limiting factor for mass exchange in 3D culture $(36,37)$. Diffusion gradients form in 3D cultures, but the cells in the center of transplants do not receive sufficient oxygen and nutrients, and eventually die (38). Increase of surface area and applying laminar flow in the 3D cultures used in conventional methods would alleviate the problem of passive diffusion and would increase cell viability. Here, we perforated the hydrogel-encapsulated quasi-natural cell blocks and used mechanically agitated cultures to generate laminar flow so that the cells within the block had more opportunity to contact oxygen and nutrients.

In this study, we successfully developed a method for high efficiency transplantation of cells in which the transplanted cells became biologically indistinguishable from 
those in the recipient tissue. Histological evaluation of quasinatural cell blocks showed that well-clustered cell lines were able to maintain proper intercellular communication, an ECM, and assemble vascular networks, allowing permanent adaptation after engraftment without immunogenicity. The transplanted quasi-natural cell block was perfectly adapted in the recipient's body; histological examination was not able to distinguish the cell block from the neighboring tissues in the recipient mice. Although the size of the quasi-natural cell block was appropriate for transplanting adherent somatic cells, it could be used as a building unit in current 3D printing technology for creation of artificial tissues or even for

\section{References}

1. Rouger K, Larcher T, Dubreil L, Deschamps JY, Le Guiner C, Jouvion G, et al. Systemic delivery of allogenic muscle stem cells induces long-term muscle repair and clinical efficacy in duchenne muscular dystrophy dogs. Am J Pathol 2011; 179: 2501-2518, doi: 10.1016/j.ajpath.2011.07.022.

2. Nardi NB, Camassola M. Isolation and culture of rodent bone marrow-derived multipotent mesenchymal stromal cells. Methods Mol Biol 2011; 698: 151-160, doi: 10.1007/978-160761-999-4_12.

3. Blurton-Jones M, Spencer B, Michael S, Castello NA, Agazaryan AA, Davis JL, et al. Neural stem cells genetically-modified to express neprilysin reduce pathology in Alzheimer transgenic models. Stem Cell Res Ther 2014; 5: 46, doi: $10.1186 /$ scrt440.

4. Wyse RD, Dunbar GL, Rossignol J. Use of genetically modified mesenchymal stem cells to treat neurodegenerative diseases. Int J Mol Sci 2014; 15: 1719-1745, doi: 10.3390/ ijms15021719.

5. Acosta SA, Franzese N, Staples M, Weinbren NL, Babilonia $\mathrm{M}$, Patel J, et al. Human umbilical cord blood for transplantation therapy in myocardial infarction. J Stem Cell Res Ther 2013 (in press).

6. Dawson E, Mapili G, Erickson K, Taqvi S, Roy K. Biomaterials for stem cell differentiation. Adv Drug Deliv Rev 2008; 60: 215228, doi: 10.1016/j.addr.2007.08.037.

7. Chatterjee K, Young MF, Simon CG Jr. Fabricating gradient hydrogel scaffolds for 3D cell culture. Comb Chem High Throughput Screen 2011; 14: 227-236, doi: 10.2174/1386207 11795222455

8. Zhu J, Marchant RE. Design properties of hydrogel tissueengineering scaffolds. Expert Rev Med Devices 2011; 8: 607-626, doi: 10.1586/erd.11.27.

9. Pramanik S, Pingguan-Murphy B, Osman NAA. Progress of key strategies in development of electrospun scaffolds: bone tissue. Sci Technol Adv Mater 2012; 13: e043002, doi: 10.1088/1468-6996/13/4/043002.

10. Baudino TA, McFadden A, Fix C, Hastings J, Price R, Borg TK. Cell patterning: interaction of cardiac myocytes and fibroblasts in three-dimensional culture. Microsc Microanal 2008; 14: 117-125, doi: 10.1017/S1431927608080021.

11. Yarlagadda PK, Chandrasekharan M, Shyan JY. Recent advances and current developments in tissue scaffolding. Biomed Mater Eng 2005; 15: 159-177.

12. Chen SS, Fitzgerald W, Zimmerberg J, Kleinman HK, creation of artificial organs. Therefore, we believe that our quasi-natural cell block technology could be widely applied for repairing damaged cells or tissues, stem cell transplantation, ex vivo gene therapy, or plastic surgery.

\section{Acknowledgments}

Research supported by the Next-Generation BioGreen 21 program, Rural Development Administration (\#PJ009528) and Technology Development Program for High ValueAdded Food, Ministry of Agriculture, Food and Rural Affairs (\#313040-03), Republic of Korea.
Margolis L. Cell-cell and cell-extracellular matrix interactions regulate embryonic stem cell differentiation. Stem Cells 2007; 25: 553-561, doi: 10.1634/stemcells.2006-0419.

13. Asakawa N, Shimizu T, Tsuda Y, Sekiya S, Sasagawa T, Yamato $\mathrm{M}$, et al. Pre-vascularization of in vitro three-dimensional tissues created by cell sheet engineering. Biomaterials 2010; 31: 3903-3909, doi: 10.1016/j.biomaterials.2010.01.105.

14. Benoit DS, Schwartz MP, Durney AR, Anseth KS. Small functional groups for controlled differentiation of hydrogelencapsulated human mesenchymal stem cells. Nat Mater 2008; 7: 816-823, doi: 10.1038/nmat2269.

15. Wong Po Foo CT, Lee JS, Mulyasasmita W, Parisi-Amon A, Heilshorn SC. Two-component protein-engineered physical hydrogels for cell encapsulation. Proc Natl Acad Sci U S A 2009; 106: 22067-22072, doi: 10.1073/pnas.0904851106.

16. Nichol JW, Khademhosseini A. Modular tissue engineering: engineering biological tissues from the bottom up. Soft Matter 2009; 5: 1312-1319, doi: 10.1039/b814285h.

17. Jurgens WJ, Oedayrajsingh-Varma MJ, Helder MN, Zandiehdoulabi B, Schouten TE, Kuik DJ, et al. Effect of tissue-harvesting site on yield of stem cells derived from adipose tissue: implications for cell-based therapies. Cell Tissue Res 2008; 332: 415-426, doi: 10.1007/s00441-0070555-7.

18. Dazzi F, van Laar JM, Cope A, Tyndall A. Cell therapy for autoimmune diseases. Arthritis Res Ther 2007; 9: 206, doi: 10.1186/ar2128.

19. Gimble JM, Katz AJ, Bunnell BA. Adipose-derived stem cells for regenerative medicine. Circ Res 2007; 100: 1249-1260, doi: 10.1161/01.RES.0000265074.83288.09.

20. Li H, Zhang B, Lu Y, Jorgensen M, Petersen B, Song S. Adipose tissue-derived mesenchymal stem cell-based liver gene delivery. J Hepatol 2011; 54: 930-938, doi: 10.1016/ j.jhep.2010.07.051.

21. Tian L, George SC. Biomaterials to prevascularize engineered tissues. J Cardiovasc Transl Res 2011; 4: 685-698, doi: 10.1007/s12265-011-9301-3.

22. Dixon JE, Shah DA, Rogers C, Hall S, Weston N, Parmenter $\mathrm{CD}$, et al. Combined hydrogels that switch human pluripotent stem cells from self-renewal to differentiation. Proc Natl Acad Sci U S A 2014; 111: 5580-5585, doi: 10.1073/pnas.1319685 111.

23. Soleimani M, Khorsandi L, Atashi A, Nejaddehbashi F. Chondrogenic differentiation of human umbilical cord blood-derived 
unrestricted somatic stem cells on A 3D beta-tricalcium phosphate-alginate-gelatin scaffold. Cell J 2014; 16: 43-52.

24. Gill RG, Wolf L. Immunobiology of cellular transplantation. Cell Transplant 1995; 4: 361-370, doi: 10.1016/09636897(95)00019-T.

25. Lu X, Liu T, Gu L, Huang C, Zhu H, Meng W, et al. Immunomodulatory effects of mesenchymal stem cells involved in favoring type 2 T cell subsets. Transpl Immunol 2009; 22: 55-61, doi: 10.1016/j.trim.2009.08.002.

26. Ahsan T, Nerem RM. Bioengineered tissues: the science, the technology, and the industry. Orthod Craniofac Res 2005; 8: 134-140, doi: 10.1111/j.1601-6343.2005.00326.x.

27. Rossello RA, Kohn DH. Gap junction intercellular communication: a review of a potential platform to modulate craniofacial tissue engineering. J Biomed Mater Res B Appl Biomater 2009; 88: 509-518, doi: 10.1002/jbm.b.31127.

28. Seal BL, Otero TC, Panitch A. Polymeric biomaterial for tissue and organ regeneration. Mater Sci Eng R Rep 2001; 34: 147-230, doi: 10.1016/S0927-796X(01)00035-3.

29. Ikada $Y$. Challenges in tissue engineering. $J R$ Soc Interface 2006; 3: 589-601, doi: 10.1098/rsif.2006.0124.

30. Doblare M, Garcia JM, Gomez MJ. Modeling bone tissue fracture and healing: a review. Eng Fract Mech 2004; 71: 1809-1840, doi: 10.1016/j.engfracmech.2003.08.003.

31. Li B, Moshfegh C, Lin Z, Albuschies J, Vogel V. Mesenchymal stem cells exploit extracellular matrix as mechanotransducer. Sci Rep 2013; 3: 2425.

32. Tibbitt MW, Anseth KS. Hydrogels as extracellular matrix mimics for 3D cell culture. Biotechnol Bioeng 2009; 103: 655-663, doi: 10.1002/bit.22361.

33. Huang $H$, Ding $Y$, Sun $X S$, Nguyen TA. Peptide hydrogelation and cell encapsulation for 3D culture of MCF-7 breast cancer cells. PLoS One 2013; 8: e59482, doi: 10.1371/journal.pone. 0059482.

34. Hollister SJ, Maddox RD, Taboas JM. Optimal design and fabrication of scaffolds to mimic tissue properties and satisfy biological constraints. Biomaterials 2002; 23: 4095-4103, doi: 10.1016/S0142-9612(02)00148-5.

35. Moore M, Sarntinoranont M, McFetridge P. Mass transfer trends occurring in engineered ex vivo tissue scaffolds. $J$ Biomed Mater Res A 2012; 100: 2194-2203, doi: 10.1002/ jbm.a.34092.

36. Glicklis R, Merchuk JC, Cohen S. Modeling mass transfer in hepatocyte spheroids via cell viability, spheroid size, and hepatocellular functions. Biotechnol Bioeng 2004; 86: 672680, doi: 10.1002/bit.20086.

37. Curcio E, Salerno S, Barbieri G, De Bartolo L, Drioli E, Bader A. Mass transfer and metabolic reactions in hepatocyte spheroids cultured in rotating wall gas-permeable membrane system. Biomaterials 2007; 28: 5487-5497, doi: 10.1016/ j.biomaterials.2007.08.033.

38. Zhang $X$, Wang W, Xie $Y$, Zhang $Y$, Wang $X$, Guo X, et al. Proliferation, viability, and metabolism of human tumor and normal cells cultured in microcapsule. Appl Biochem Biotechnol 2006; 134: 61-76, doi: 10.1385/ABAB:134: 1:61. 